\title{
Identification of mistuning and model updating of an academic blisk based on geometry and vibration measurements
}

\author{
F. Nyssen ${ }^{\mathrm{a}, *}$, J.-C. Golinval ${ }^{\mathrm{a}}$ \\ ${ }^{a}$ University of Liege, Department of Aerospace and Mechanical Engineering, \\ Allee de la Decouverte 9, 4000 Liege, Belgium
}

\begin{abstract}
In this work, an experimental modal analysis is performed on an academic bladed disk using a base excitation to identify the mistuning of each blade. Optical measurement is used to obtain the exact geometry of the structure and to be able to associate geometrical mistuning to each blade. Differences are observed between the experimentally identified mistuning and the geometrical mistuning. Since the bladed disk is a onepiece structure, there is no welded connections between the blades and the disk and the material properties can be assumed to be uniform. It can be shown that these differences come from non uniform clamping conditions, and that this mistuning is of the same order of magnitude than the variations in the geometry of the structure. It follows that the precise characterisation of mistuning for industrial structures is in practice illusory because of the numerous factors introducing mistuning, such as the clamping conditions, aerodynamic damping, wear in service, etc.
\end{abstract}

Keywords: Bladed structure, mistuning, experimental modal identification, geometry measurement, model updating

\section{Nomenclature:}

$\gamma \quad$ Damping coefficient

$\lambda_{i, r} \quad$ Eigenvalue deviation of the $r^{\text {th }}$ cantilevered blade mode of blade $i$

$\boldsymbol{\Lambda}_{0} \quad$ Generalized stiffness matrix of the tuned system

$\Lambda^{C B} \quad$ Generalized stiffness matrix of the cantilevered blade

$\Phi \quad$ Tuned mode-shapes matrix

$\boldsymbol{\Phi}_{i} \quad$ Tuned mode-shapes matrix restricted to the degrees of freedom of blade $i$

$\boldsymbol{\Phi}_{i}^{M} \quad$ Mistuned mode-shapes matrix restricted to the degrees of freedom of blade $i$

$\boldsymbol{\Phi}^{C B} \quad$ Cantilevered blade modes for the nominal blade

$\boldsymbol{\Phi}_{\text {exp }} \quad$ Tuned displacements of the measured degrees of freedom

$\omega \quad$ Frequency

\footnotetext{
* Corresponding author

Email address: Florence.Nyssen@ulg.ac.be (F. Nyssen)
} 


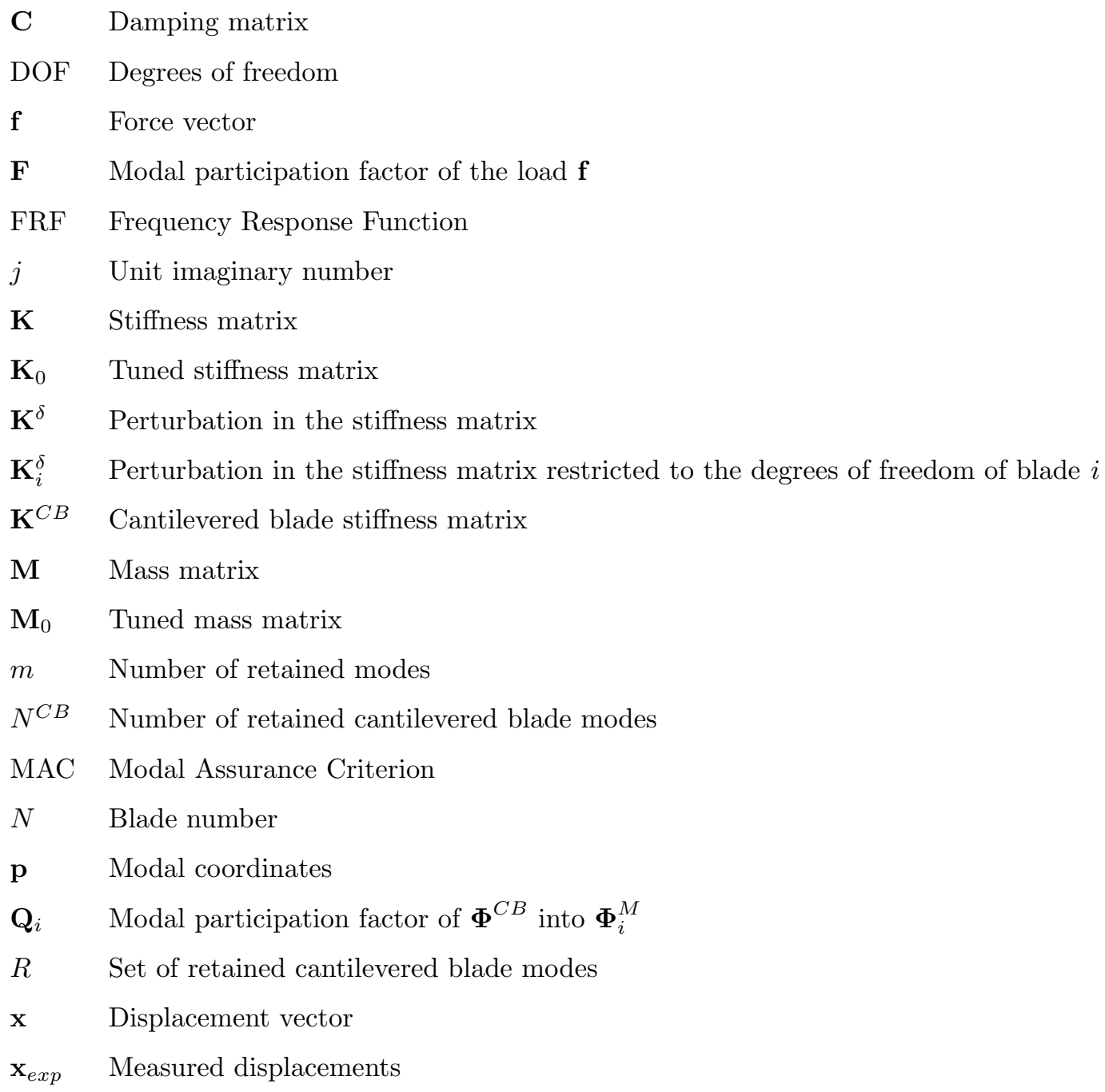

\section{Introduction}

In the field of turbomachinery, various assumptions are usually made to model bladed assemblies, such as cyclic symmetry of rotor stages. This assumption enables, in a linear framework, to reduce drastically the required computational resources by considering one sector instead of the entire assembly. However,

5 even if the bladed disks are designed to be cyclically symmetric, small random blade-to-blade variations appear in practice due to manufacturing tolerances, irregularities in the material properties, wear during use, etc. These small variations, known as mistuning, disturb dramatically the dynamic behavior of bladed disks (energy localization in only few blades, amplification of the forced response) [1].

Different reduced-order modeling techniques have been developed to compute the dynamic behavior of mistuned bladed disks with reduced computational time [2, 3, 4, 5, 6, 7, 8, 9, 10, 11, 12, 13, 14. These reduced-order models (ROMs) enable to construct low-order models of bladed disks based on the global 
finite element model of the structures. Using ROMs enables to run a large number of simulations with random mistuning patterns in order to retrieve the statistical distribution of the amplification of the forced response amplitudes for given amounts of mistuning.

In parallel, identification methods have been developed, to identify mistuning from the experimental frequency response of bladed structure [15, 16, 17, 13. A usual assumption consists in proportional mistuning i.e., the variation of each blade is considered to be proportional to the stiffness of the tuned sector. First studies tested separately each individual blade to determine their deviation from the nominal design $[8,15]$. However, additional mistuning is introduced when blades are assembled on the disk. Moreover, many bladed disks are now manufactured in one-piece, known as integrally bladed disks or blisks. Several methods have been developed to identify mistuning from measurements of the response of the entire bladed disk rather than on individual and isolated blades. Early works were based on lumped parameter models in order to identify the structural parameters of blades [18, 19. More recently, identification methods based on ROMs have been developed [20, 21, 16, 17, 22, 23, 24, 25].

The component mode mistuning (CMM) method developed by Lim [13] is used in this work, in which the mistuning identified for each blade is represented as the variation of the cantilevered blade frequency. An experimental modal analysis is performed here on a mono-stage academic blisk in order to apply Lim's mistuning identification method. The frequency response function (FRF) is measured in terms of sensitivity using a base excitation with an electrodynamic shaker and one measurement point per blade is acquired at the tip of each blade with a laser vibrometer.

The exact geometry of the academic structure is then measured by means of an optical geometry measurement system, enabling to quantify the level of geometric mistuning [26. Based on these optical data, a finite element model of the structure is constructed and the FRFs of this model are compared to the experimental FRFs.

This enables to quantify the relative importance of geometric mistuning compared to other sources of mistuning [27. In this case, since the studied bladed disk is a one-piece structures, there is no welded connections between the blades and the disk and the material properties can be assumed to be uniform. Thus, the main source of additional mistuning for this structure is the clamping condition which can not be perfect in practice.

\section{Component mode mistuning (CMM) method}

Lim 13$]$ developed in his thesis two distinct approaches: a general method involving large models and potentially long computation times, and a simplified version of this method based on the work of Yang and Griffin [28] and Bladh [7]. The second approach, which is known as Component Mode Mistuning (CMM) method, is used in this work. 
This simplified approach may be considered assuming that the blade mistuning is small compared to nominal properties in the modal domain, and that the bladed disk has normal modes closely spaced in a frequency range. The second assumption means that the tuned modes outside of the frequency range of interest can be ignored in modelling the mistuned system.

The theoretical developments of CMM method are reminded for consistency. The equations of motion in the physical space are given by

$$
\mathbf{M} \ddot{\mathbf{x}}+\mathbf{C} \dot{\mathbf{x}}+\mathbf{K x}=\mathbf{f}
$$

where $\mathbf{M}, \mathbf{C}$ and $\mathbf{K}$ are respectively the mass, damping and stiffness matrices, $\mathbf{x}$ is the displacement vector, and $\mathbf{f}$ is the force vector. Equation (1) can be projected in the modal space using

$$
\mathbf{x}=\mathbf{\Phi} \mathbf{p}
$$

where $\boldsymbol{\Phi}$ is the tuned mode-shapes matrix of the system (the number of retained modes in the $\boldsymbol{\Phi}$ matrix is noted $m$ ), and $\mathbf{p}$ is the modal coordinates vector:

$$
\boldsymbol{\Phi}^{T} \mathbf{M} \boldsymbol{\Phi} \ddot{\mathbf{p}}+\boldsymbol{\Phi}^{T} \mathbf{C} \boldsymbol{\Phi} \dot{\mathbf{p}}+\boldsymbol{\Phi}^{T} \mathbf{K} \boldsymbol{\Phi} \mathbf{p}=\boldsymbol{\Phi}^{T} \mathbf{f}
$$

or in the frequency domain, and considering a modal damping ( $\gamma$ is the damping coefficient),

$$
-\omega^{2} \boldsymbol{\Phi}^{T} \mathbf{M} \boldsymbol{\Phi} \mathbf{p}+(1+j \gamma) \boldsymbol{\Phi}^{T} \mathbf{K} \boldsymbol{\Phi} \mathbf{p}=\boldsymbol{\Phi}^{T} \mathbf{f}
$$

The mistuning is then introduced in the equations of motion. A small mistuning is considered here i.e. the perturbation of the nominal modal properties of the system is small. This implies that the mistuned mode-shapes can be expressed as a combination of tuned mode-shapes in the frequency range of interest. Assuming that the mistuning is represented by a stiffness modification, the mistuned equations of motion can be written as

$$
-\omega^{2} \boldsymbol{\Phi}^{T} \mathbf{M}_{0} \boldsymbol{\Phi} \mathbf{p}+(1+j \gamma) \boldsymbol{\Phi}^{T}\left(\mathbf{K}_{0}+\mathbf{K}^{\delta}\right) \boldsymbol{\Phi} \mathbf{p}=\boldsymbol{\Phi}^{T} \mathbf{f}
$$

in which $\mathbf{M}_{0}$ and $\mathbf{K}_{0}$ represent respectively the tuned mass and stiffness matrix, and $\mathbf{K}^{\delta}$ represents the perturbation in the stiffness matrix due to mistuning. Then, considering that the mode-shapes are massnormalized, equation (5) becomes

$$
-\omega^{2} \mathbf{I} \mathbf{p}+(1+j \gamma)\left[\boldsymbol{\Lambda}_{0}+\boldsymbol{\Phi}^{T} \mathbf{K}^{\delta} \mathbf{\Phi}\right] \mathbf{p}=\mathbf{F}
$$

where $\mathbf{I}$ is the identity matrix, $\boldsymbol{\Lambda}_{0}$ is the generalized stiffness matrix, and posing $\mathbf{F}=\boldsymbol{\Phi}^{T} \mathbf{f}$. 
Then, three assumptions are performed in CMM. First, the mistuning is considered to be located on the blades only and not on the disk, since a mistuning located on the blades is known to have a larger impact than disk mistuning. This implies that matrix $\mathbf{K}^{\delta}$ is non-zero only in the blade portion i.e.,

$$
\boldsymbol{\Phi}^{T} \mathbf{K}^{\delta} \boldsymbol{\Phi}=\sum_{i=1}^{N} \boldsymbol{\Phi}_{i}^{T} \mathbf{K}_{i}^{\delta} \boldsymbol{\Phi}_{i}
$$

where $N$ is the number of blades, $\mathbf{K}_{i}^{\delta}$ is the stiffness matrix restricted to the degrees of freedom of blade $i$ $(i=1, \ldots, N)$, and $\boldsymbol{\Phi}_{i}$ is the matrix of tuned mode-shapes restricted to the degrees of freedom of blade $i$.

The second assumption in CMM is that the mode-shape matrix $\boldsymbol{\Phi}_{i}$ of blade $i$ is written as a superposition of cantilevered blade modes i.e.,

$$
\boldsymbol{\Phi}_{i}=\boldsymbol{\Phi}^{C B} \mathbf{Q}_{i}
$$

in which $\boldsymbol{\Phi}^{C B}$ is the cantilevered blade modes for the nominal blade and $\mathbf{Q}_{i}$ is the modal participation factor of $\boldsymbol{\Phi}^{C B}$ into the mistuned mode-shape of the blade's degrees of freedom $\boldsymbol{\Phi}_{i}^{M}$. Introducing equation (8) in equation (7) leads to

$$
\boldsymbol{\Phi}^{T} \mathbf{K}^{\delta} \boldsymbol{\Phi}=\sum_{i=1}^{N} \mathbf{Q}_{i}^{T} \boldsymbol{\Phi}^{C B^{T}} \mathbf{K}_{i}^{\delta} \boldsymbol{\Phi}^{C B} \mathbf{Q}_{i}
$$

The expression $\boldsymbol{\Phi}^{C B^{T}} \mathbf{K}_{i}^{\delta} \boldsymbol{\Phi}^{C B}$ in equation $(9)$ has off-diagonal terms. However, one tuned cantilevered blade mode is usually dominant if mistuning is small. Therefore, the expression $\boldsymbol{\Phi}^{C B^{T}} \mathbf{K}_{i}^{\delta} \boldsymbol{\Phi}^{C B}$ is diagonally dominant and the off-diagonal terms, which represent the coupling between cantilevered blade modes, can be neglected. This third assumption enables to simplify equation (9) as

$$
\boldsymbol{\Phi}^{T} \mathbf{K}^{\delta} \boldsymbol{\Phi}=\sum_{i=1}^{N} \mathbf{Q}_{i}^{T} \operatorname{diag}_{r \in R}\left(\lambda_{i, r}\right) \mathbf{Q}_{i}
$$

where $R$ is the set of retained cantilevered blade modes and $\lambda_{i, r}=\boldsymbol{\Phi}_{r}^{C B^{T}} \mathbf{K}_{i}^{\delta} \boldsymbol{\Phi}_{r}^{C B}$ is the eigenvalue deviation

of the $r^{t h}$ cantilevered blade mode of blade $i$. Introducing equation $(10$ in the equation (6), we have

$$
-\omega^{2} \mathbf{I} \mathbf{p}+(1+j \gamma)\left[\boldsymbol{\Lambda}_{0}+\sum_{i=1}^{N} \mathbf{Q}_{i}^{T} \operatorname{diag}_{r \in R}\left(\lambda_{i, r}\right) \mathbf{Q}_{i}\right] \mathbf{p}=\mathbf{F}
$$

The computation of the participation factors $\mathbf{Q}_{i}$ in equation 11 is performed by rewriting equation (8). First, equation $(8)$ is pre-multiplied by $\boldsymbol{\Phi}^{C B^{T}} \mathbf{K}^{C B}$, such as

$$
\boldsymbol{\Phi}^{C B^{T}} \mathbf{K}^{C B} \boldsymbol{\Phi}_{i}=\boldsymbol{\Phi}^{C B^{T}} \mathbf{K}^{C B} \boldsymbol{\Phi}^{C B} \mathbf{Q}_{i}
$$




$$
\boldsymbol{\Phi}^{C B^{T}} \mathbf{K}^{C B} \boldsymbol{\Phi}_{i}=\Lambda^{C B} \mathbf{Q}_{i}
$$

where $\Lambda^{C B}$ is a diagonal matrix containing the squared eigenfrequencies of the cantilevered blade modes.

Finally, the expression of the participation factor $\mathbf{Q}_{i}$ can be written as

$$
\mathbf{Q}_{i}=\Lambda^{C B^{-1}} \boldsymbol{\Phi}^{C B^{T}} \mathbf{K}^{C B} \mathbf{\Phi}_{i}
$$

Using the two equations (11) and (14), the mistuned properties of the system can be computed for a given mistuning pattern. All the needed input data can be retrieved from two finite element models:

- the finite element model of the tuned structure, which enables to retrieve the tuned mode-shapes restricted to the blades degrees of freedom, $\boldsymbol{\Phi}_{i}$, and the eigenvalues of the tuned system in order to obtain $\boldsymbol{\Lambda}_{0}$.

- and the finite element model of the blade clamped at its basis, which enables to compute the cantilevered blade eigenvalues of in order to construct $\Lambda^{C B}$, the cantilevered blade modes $\boldsymbol{\Phi}^{C B}$, and the cantilevered blade stiffness matrix $\mathbf{K}^{C B}$.

Reorganizing the terms of equation (11), we obtain

$$
\sum_{i=1}^{N} \mathbf{Q}_{i}{ }^{T} \operatorname{diag}_{r \in R}\left(\lambda_{i, r}\right) \mathbf{Q}_{i} \mathbf{p}=\frac{1}{(1+j \gamma)}\left[\mathbf{F}+\omega^{2} \mathbf{I} \mathbf{p}-(1+j \gamma) \boldsymbol{\Lambda}_{0} \mathbf{p}\right] .
$$

Equation 15 is linear in $\lambda_{i, r}$. The number of unknowns is equal to the number of blades $N$ multiplied by the number of retained cantilevered blade modes $N^{C B}$. Considering that $m$ modes are measured, equation 15 can be written as

$$
\begin{array}{ccccc}
{\left[\begin{array}{ccccc}
\mathbf{Q}_{1,1}{ }^{T} \mathbf{Q}_{1,1} \mathbf{p}_{1} & \mathbf{Q}_{1,2}{ }^{T} \mathbf{Q}_{1,2} \mathbf{p}_{1} & \ldots & \mathbf{Q}_{N, N^{C B}}{ }^{T} \mathbf{Q}_{N, N^{C B}} \mathbf{p}_{1} \\
\mathbf{Q}_{1,1}{ }^{T} \mathbf{Q}_{1,1} \mathbf{p}_{2} & \mathbf{Q}_{1,2}{ }^{T} \mathbf{Q}_{1,2} \mathbf{p}_{2} & \ldots & \mathbf{Q}_{N, N^{C B}}{ }^{T} \mathbf{Q}_{N, N^{C B}} \mathbf{p}_{2} \\
\vdots & & \ldots & \vdots \\
\mathbf{Q}_{1,1}{ }^{T} \mathbf{Q}_{1,1} \mathbf{p}_{m} & \mathbf{Q}_{1,2}{ }^{T} \mathbf{Q}_{1,2} \mathbf{p}_{m} & \ldots & \mathbf{Q}_{N, N^{C B}}{ }^{T} \mathbf{Q}_{N, N^{C B}} \mathbf{p}_{m}
\end{array}\right]\left[\begin{array}{c}
\lambda_{1,1} \\
\lambda_{1,2} \\
\vdots \\
\lambda_{N, N^{C B}}
\end{array}\right]=} \\
\frac{1}{(1+j \gamma)} \\
{\left[\begin{array}{c}
\mathbf{F}_{1}+\omega_{1}^{2} \mathbf{p}_{1}-(1+j \gamma) \mathbf{\Lambda}_{0} \mathbf{p}_{1} \\
\mathbf{F}_{2}+\omega_{2}^{2} \mathbf{p}_{2}-(1+j \gamma) \Lambda_{0} \mathbf{p}_{2} \\
\vdots \\
\mathbf{F}_{m}+\omega_{m}^{2} \mathbf{p}_{m}-(1+j \gamma) \mathbf{\Lambda}_{0} \mathbf{p}_{m}
\end{array}\right]}
\end{array}
$$


where $\mathbf{p}_{k}, \mathbf{F}_{k}$ and $\omega_{k}$, with $k=1, \ldots, m$, are the data related to the $k^{t h}$ measured mode, and $\mathbf{Q}_{i, r}$ refers to the $r^{t h}$ lines of $\mathbf{Q}_{i}$. Moreover, if the frequencies $\omega_{k}(k=1, \ldots, m)$ are chosen to be the eigenfrequencies of the system, the force vector $\mathbf{F}_{k}$ can be neglected. This group of equations can be divided in a set of real equations and a set of imaginary equations. Since the mistuning parameters are real, they can be obtained by solving both set of equations.

\section{Experimental test procedure}

A simple test procedure is established, based on equation (16) of the CMM method.

1. A base excitation is used to excite the structure in the frequency range of interest. The response of the structure is measured on each blade with a laser vibrometer.

2. A stabilization diagram is constructed based on the FRF measured on each blade in terms of transmissibility.

3. The eigenfrequencies and mode-shapes can be retrieved using the stabilization diagram.

4. The modal coordinates $\mathbf{p}$ are computed based on the measured physical displacements $\mathbf{x}_{e x p}$ as

$$
\mathbf{x}_{\exp }=\boldsymbol{\Phi}_{\exp } \mathbf{p}
$$

where $\boldsymbol{\Phi}_{e x p}$ is a matrix that contains the tuned displacements of the measured degrees of freedom. If the matrix $\boldsymbol{\Phi}_{\exp }$ is not a square matrix (i.e. if the number of measured degrees of freedom per blade is larger than $m * N^{C B}$ ), the modal coordinates $\mathbf{p}$ are obtained by solving the least squares problem.

5. Since $\omega$ and $\mathbf{p}$ are known from the experimental tests, the unknowns in equation (16) are the $\lambda_{i, r}$ and the mistuning can be computed.

\section{Presentation of the academic structure}

The structure considered here is an academic blisk of 24 sectors made of aluminum (Fig. 1(a)). It is clamped at its basis as shown in Fig. 1(b) such that the boundary conditions respect the cyclic symmetry property. The characteristics are given in Table 1, where the Young's modulus was determined experimentally.

In this example, the mistuning can be assumed to be small. Actually, the structure has a simplified geometry and the manufacturing tolerances are indeed of the order of $0.1 \mathrm{~mm}$ for blade length of $90 \mathrm{~mm}$, which results in a few percents of blade mistuning. This enables to satisfy the assumption of CMM.

A finite element model of the tuned structure has been constructed using the FE software LMS SAMTECH SAMCEF [29]. The cyclic symmetry property has been applied in order to reduce the computational time and resources. The finite element model, with its reference sector in dark gray, is shown in Fig. 1(a). The mesh totalizes 8496 degrees of freedom per sector. 


\begin{tabular}{c|c} 
Number of sectors $(N)[-]$ & 24 \\
Young's modulus $[\mathrm{MPa}]$ & 72340 \\
Density $\left[\mathrm{kg} / \mathrm{m}^{3}\right]$ & 2700 \\
External diameter $[\mathrm{mm}]$ & 420 \\
Internal diameter $[\mathrm{mm}]$ & 186 \\
Thickness $[\mathrm{mm}]$ & 5 \\
Heigh $[\mathrm{mm}]$ & 53
\end{tabular}

Table 1: Material and geometric properties of the academic structure.

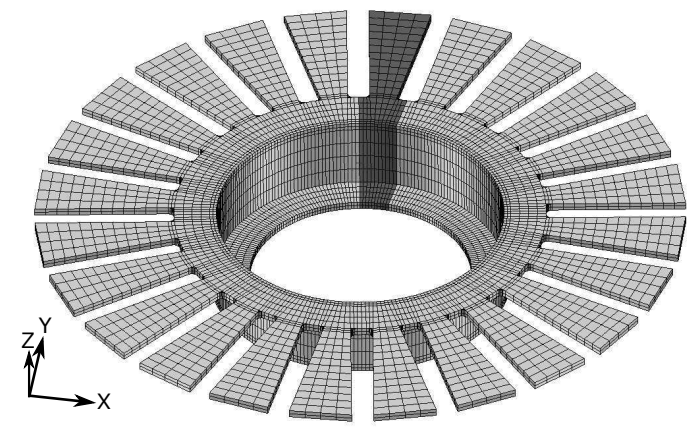

(a)

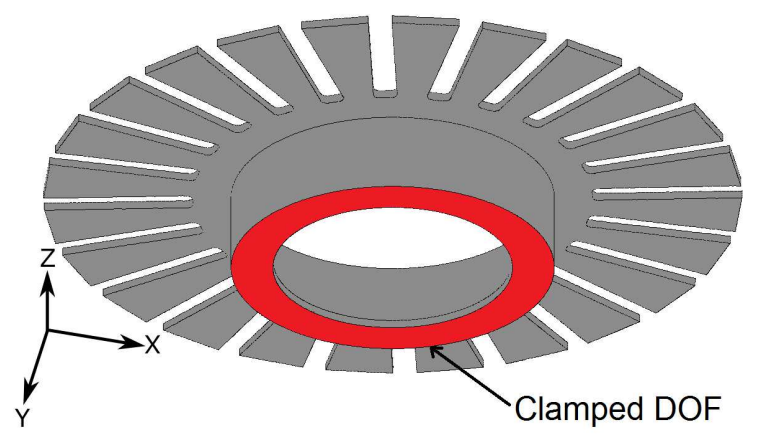

(b)

Figure 1: Full (360 degrees) finite element model of the academic structure with the reference sector in dark gray (a) and clamping conditions (b).

Based on the finite element model, the SAFE diagram (Singh's Advanced Frequency Evaluation diagram i.e., the eigenfrequencies as a function of the number of nodal diameters), sometimes referred to as veering diagram, is constructed. The maximum number of nodal diameters is $\frac{N}{2}=12$. Fig. 2 shows the SAFE diagram restricted to the first family of mode i.e., the first bending modes of the blades (1B modes). The first family of modes is chosen in this work to apply Lim's identification method because they respond at low frequencies, so that they are more easily experimentally excited with a global excitation (base excitation with a shaker), and this family is well separated from the other ones.

The range of frequency is $240 \mathrm{~Hz}$ (0 nodal diameter mode) to $320 \mathrm{~Hz}$ (12 nodal diameters mode). Despite the fact that the modal density seems to be low in comparison to what may be observed on industrial blisks, the CMM method still can be applied because only perturbation in the stiffness matrix is considered and the considered family is well isolated.

Then the clamped blade eigenfrequencies are computed. These are obtained by clamping the degrees of freedom of the disk, as shown in Fig. 3 . Only the first mode i.e., the 1B mode, is needed here. It has a frequency of $407 \mathrm{~Hz}$. 


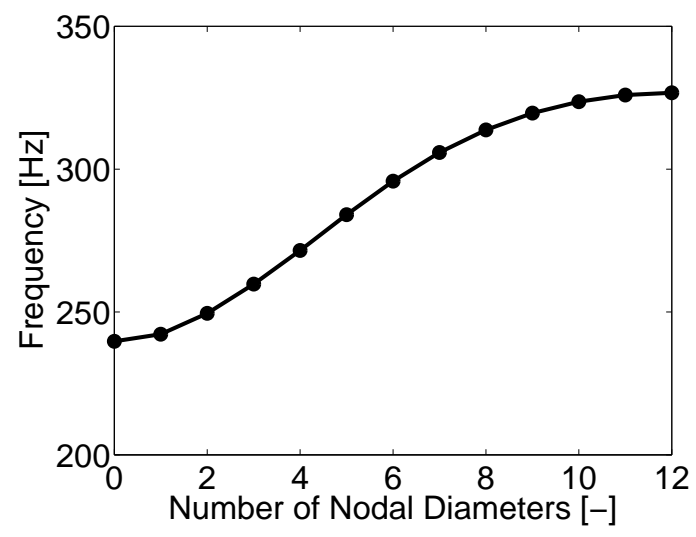

Figure 2: SAFE diagram of the academic structure restricted to the $1 \mathrm{~B}$ mode family.

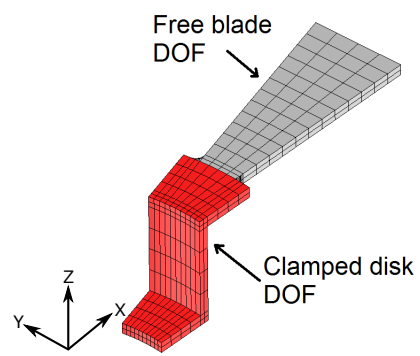

Figure 3: Clamped sector.

\section{Experimental vibration measurements}

Experimental vibration tests on the academic structure are performed in order to retrieve mistuning of each blade. The CMM method is applied to this purpose.

\subsection{Test set-up}

The academic structure and the test set-up are presented in Fig. 4 . The structure is clamped at its basis with 24 fixation points (as shown in Fig. 5) in order to keep the cyclic symmetry: the academic structure has 24 sectors and one fixation point per sector has been considered. A base excitation is applied using an electrodynamic shaker in the axial direction (Z-axis). A laser vibrometer is used to measure the response of the structure. An accelerometer measures the acceleration of the shaker in order to have the force injected by the shaker. A periodic chirp is used to excite the structure, with a resolution of $0.02 \mathrm{~Hz}$. The frequency range is 200 to $400 \mathrm{~Hz}$ i.e., the 1B modes. One measurement point is considered on each blade tip. 


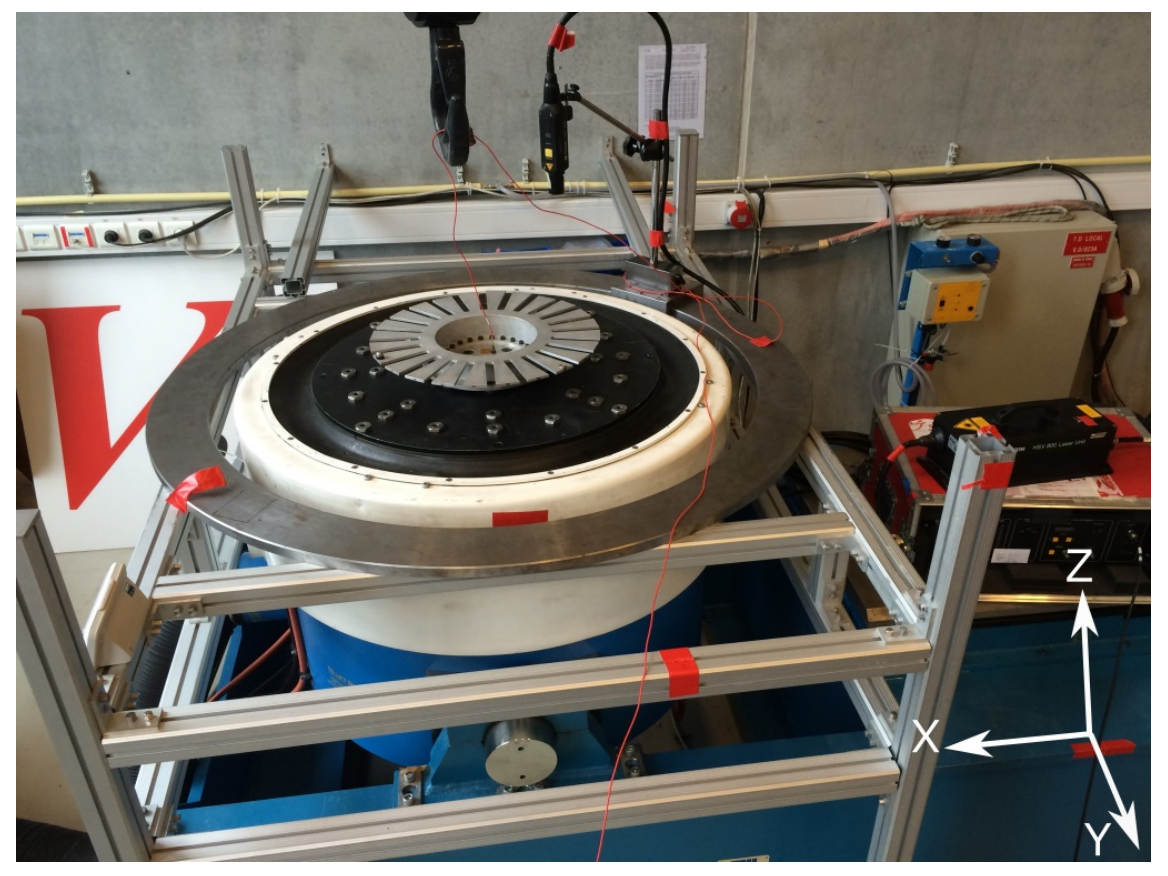

Figure 4: Academic structure and test set-up.

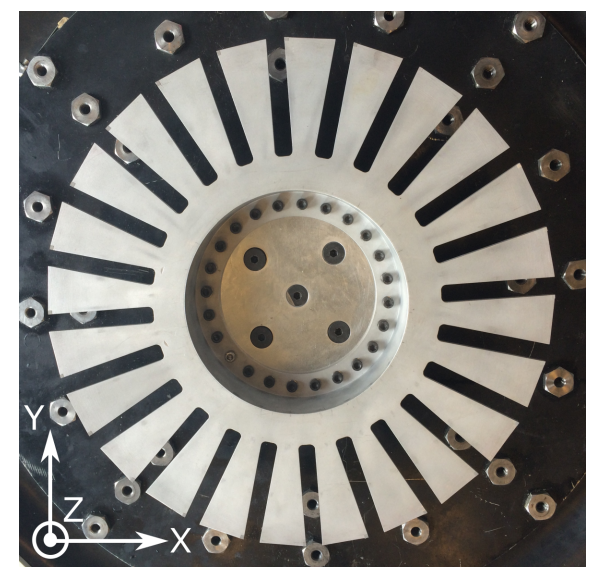

Figure 5: Clamping of the structure with 24 fixation points.

\subsection{Mistuning identification}

Figure 6 presents the stabilization diagram obtained using the PolyMax method [30] available in the LMS Test.Lab software [31]. The frequency band is [240-340] Hz, i.e. including all the peaks corresponding to 1B modes. The obtained FRF for all measurement points are superimposed. For each peak, a corresponding frequency and mode-shape is computed with the PolyMax method. The frequencies of the all 1B modes are given in Table 2 . 


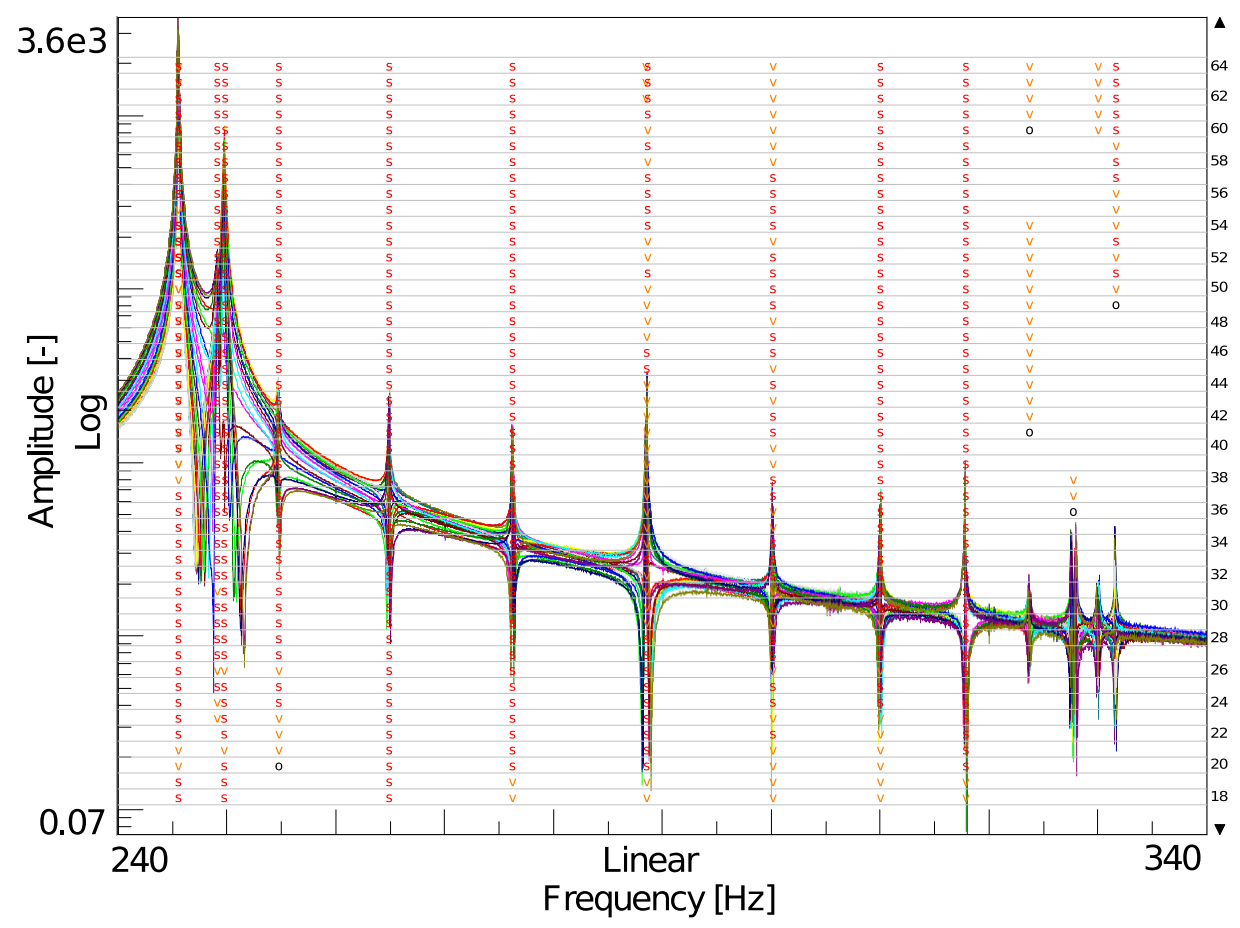

Figure 6: Stabilization diagram: 1B modes.

\begin{tabular}{c|c} 
Frequency $[\mathrm{Hz}]$ & Main harmonic [-] \\
\hline 245.5 & 0 \\
$249.1-249.7$ & 1 \\
254.7 & 2 \\
264.9 & 3 \\
276.2 & 4 \\
288.6 & 5 \\
300.1 & 6 \\
310.0 & 7 \\
317.8 & 8 \\
323.7 & 9 \\
$327.5-328.0$ & 10 \\
330.0 & 11 \\
331.6 & 12
\end{tabular}

Table 2: Eigenfrequencies of the 1B modes. 
The three 1B modes used for the identification process are the three first modes, at a frequency of 245.5, 249.1 and $249.7 \mathrm{~Hz}$ respectively, which are the most excited using the base excitation. Their mode-shapes

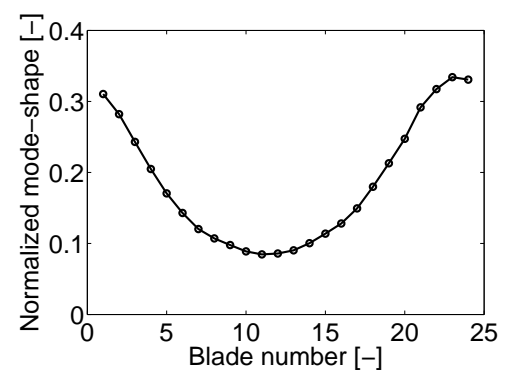

(a)

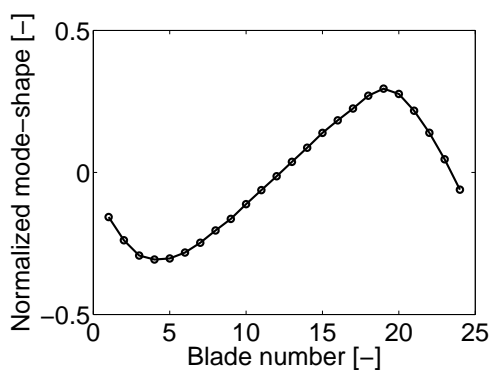

(b)

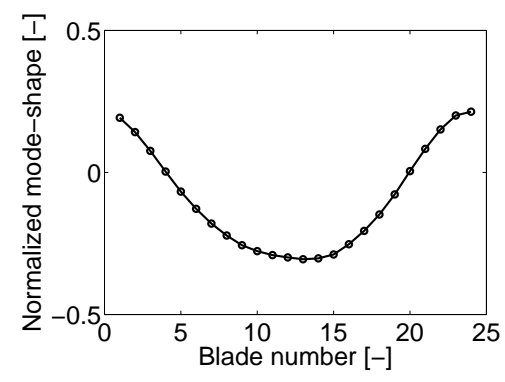

(c)

Figure 7: Mode-shapes corresponding to the peaks at a frequency of $245.5 \mathrm{~Hz}$ (a), $249.1 \mathrm{~Hz}$ (b) and $249.7 \mathrm{~Hz}$ (c).

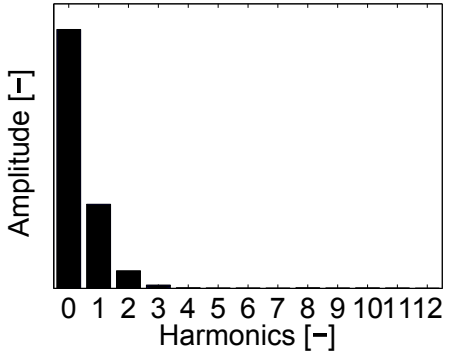

(a)

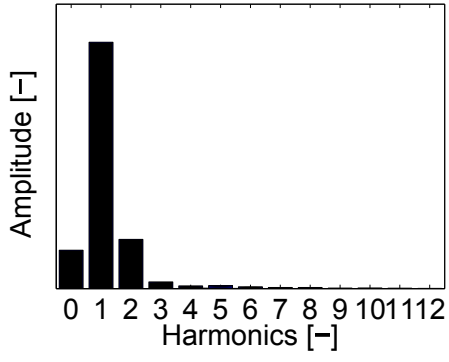

(b)

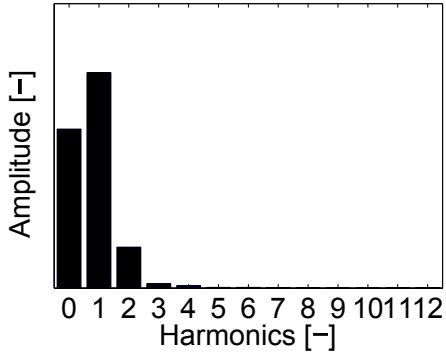

(c)

Figure 8: Harmonic content of the mode-shapes corresponding to the peaks at a frequency of $245.5 \mathrm{~Hz}$ (a), $249.1 \mathrm{~Hz}$ (b) and $249.7 \mathrm{~Hz}$ (c). 
The CMM identification method is applied using the three first experimental modes as input data. The obtained mistuning for each blade of the academic structure is presented in Fig. 9 . A mistuning up to 7\% is found for blade number 12. These identified values are used in the following sections to correlate a numerical model with the experimental results on the academic structure.

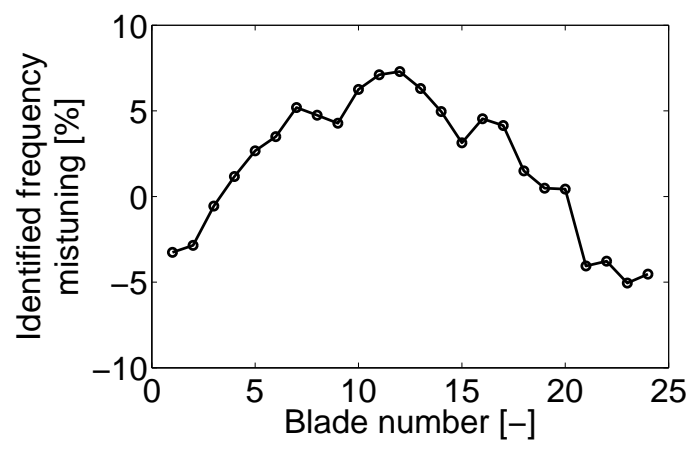

Figure 9: Identified mistuning.

\section{Optical measurement of the geometry}

The geometry of the structure is measured with a 3D camera 32. The aim of these measurements is to compare the mistuning due to changes in geometry induced by the manufacturing tolerances, with the mistuning identified using vibration tests.

\subsection{Test set-up}

An optical geometry measurement system is used to obtain the geometry of each blade. The test set-up is shown in Fig. 10(a). The optical system is composed of a central Light Emitting Diode (LED) source to illuminate the structure with a regular grid pattern projection. The distorted profile of the structure is captured by two cameras on either side of the LED source and the projected shape is used to triangulate surface coordinates. A series of picture are captured and merged together using common reference points. These standard reference circles placed on the structure are shown in Fig. 10(b). The geometry of the structure can be retrieved with an accuracy of $50 \mu m[26$. 


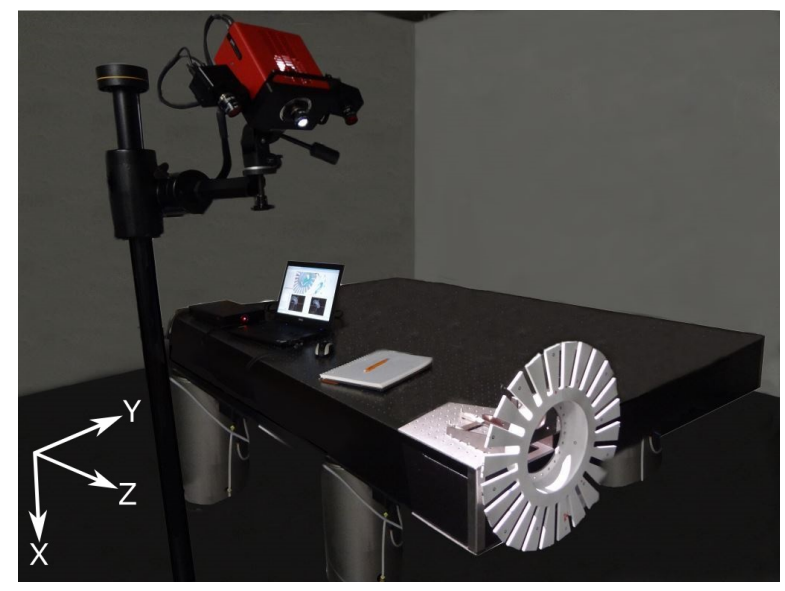

(a)

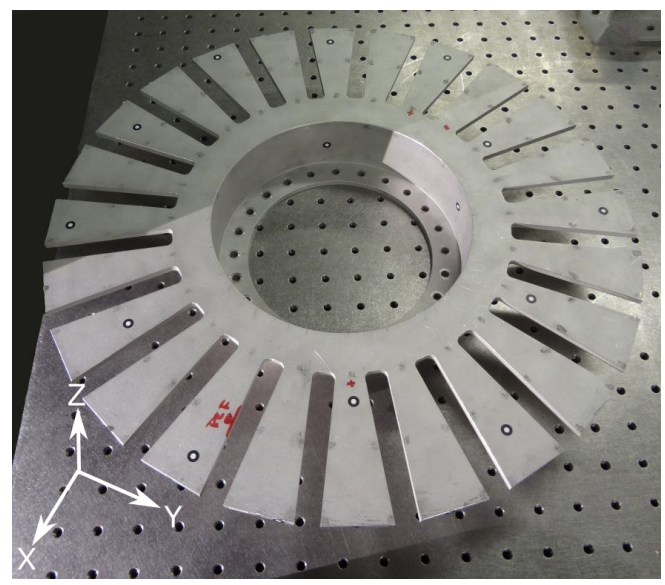

(b)

Figure 10: Test set-up for the optical measurement of the geometry (a) and standard reference circles placed on the structure (b).

Figures 11(a) and 11(b) show the precise profile of the upper and lower face of the structure respectively. The profile is defined as the vertical deviation of the measured geometry compared to a horizontal plane at an elevation of 0 and $-5 \mathrm{~mm}$ respectively for the upper and lower face. The upper bound (red color) represents the maximum vertical elevation of the points of the geometry compared to a theoretical plane (the plane of the reference tuned structure), and the lower bound (blue color) is the minimum value of the elevation. A positive sign represents a point at higher vertical coordinate compared to its theoretical position, and a negative sign represents a lower vertical coordinate of the point compared to its theoretical position. The black curve on the left of the color bar shows the repartition of the elevation, between $-0.08 \mathrm{~mm}$ and $0.08 \mathrm{~mm}$ for the upper surface. The middle bound (green color) represents the theoretical value. The majority of the values are between $0.02 \mathrm{~mm}$ and $-0.08 \mathrm{~mm}$. For the lower surface, the scale is between $-0.08 \mathrm{~mm}$ and $0.1 \mathrm{~mm}$. The middle bound (green color) represents the theoretical value. The majority of the values are between $0.02 \mathrm{~mm}$ and $-0.08 \mathrm{~mm}$.

\subsection{Construction of the finite element model}

Based on the optical geometry measurement, a refined finite element model of the structure is constructed. In this finite element model, each sector has different shapes, the Young's modulus remaining uniform for all the structure. The data given by the optical geometry measurement system is a set of points of the envelope of the structure. This set of points is presented in Fig. 12. The initial tuned mesh (i.e. the mesh with exactly identical sectors) is modified by adapting the elevation of each node to follow the profile measured by the optical system. 


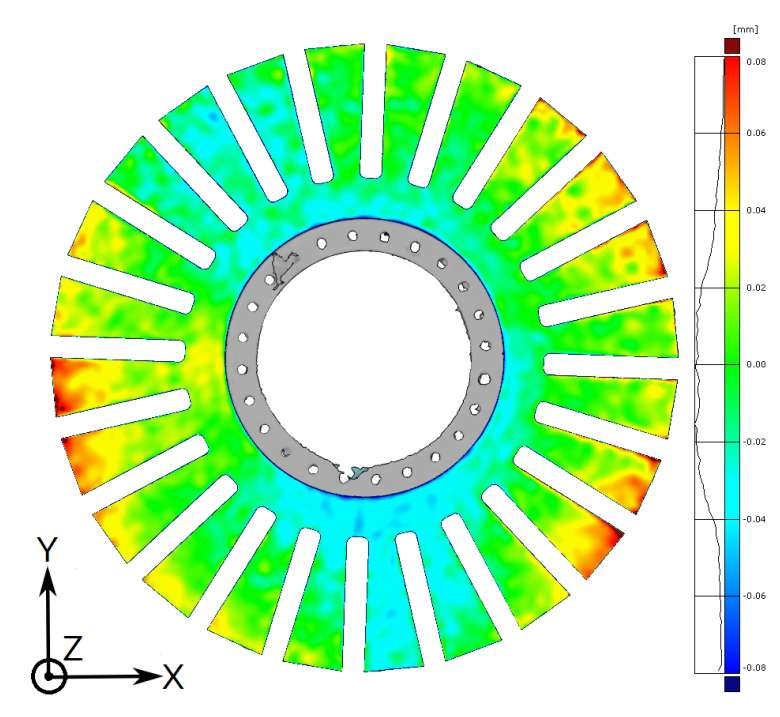

(a)

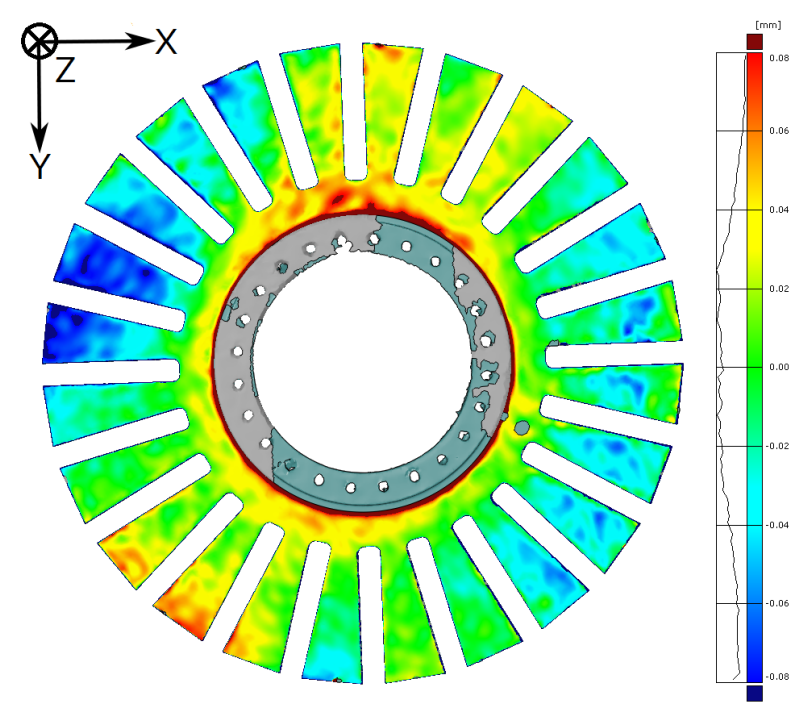

(b)

Figure 11: Measured profile of the upper (a) and lower (b) face of the structure.

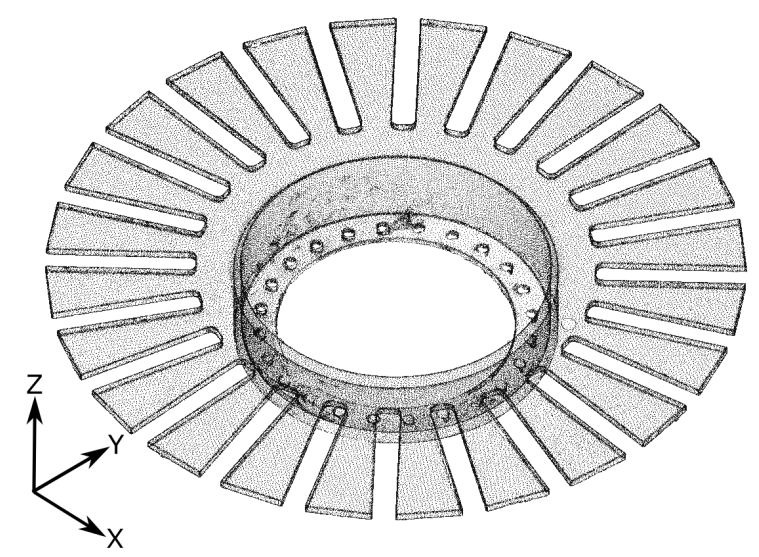

Figure 12: Set of points of the envelope of the structure given by the optical measurement system.

The eigenfrequencies and the corresponding mode-shapes are computed for the refined finite element model. Figure 13 shows the three first mode-shapes (one point per sector is considered, at the tip of each blade). The mode-shapes are normalized using the modal scale factor (MSF). Large differences are observed between both mode-shapes. The MAC number is 0.9 for the first mode, and 0.7 for the second and third modes but in a different order. Different sources can explain these differences, such as variations in the material properties, differences in the boundary conditions (in the finite element model, a perfect clamping of the structure is assumed, but this assumption is not feasible in practice), ... 


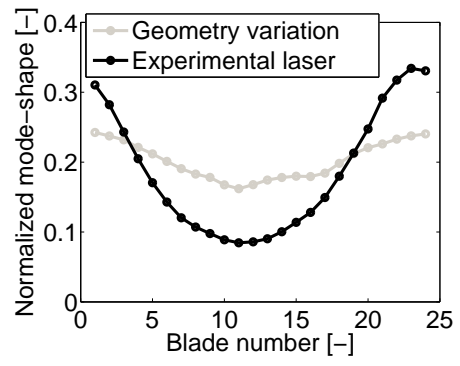

(a)

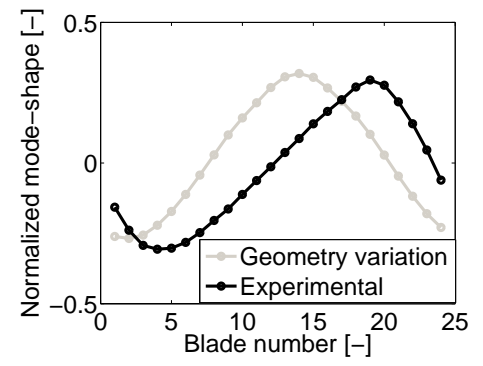

(b)

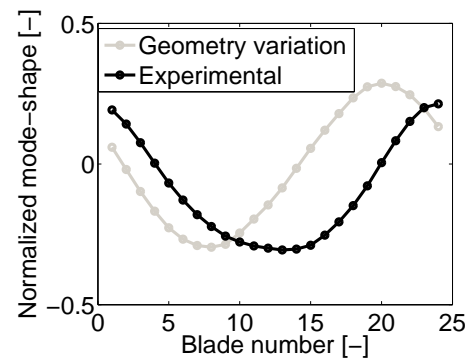

(c)

Figure 13: Mode-shapes corresponding to the first (a), to the second (b) and to the third eigenfrequency (c).

\section{Comparison of the frequency and geometric mistuning and update of the clamping condi- tions}

In order to update the model and to better match with the experimental data in terms of mode-shapes, the clamped blade eigenfrequencies of our model are compared to the mistuning identified experimentally, as shown in Fig. 14. Differences are observed. Since the studied bladed disk is a one-piece structure, there are no welded connections between the blades and the disk and the material properties can be assumed to be uniform. The main remaining source of mistuning is the boundary conditions. Indeed, in the finite element model, a perfect and uniform clamping of the structure is assumed, but this assumption is not feasible in practice. In order to update the finite element model, the perfect clamping condition is replaced by springs, with a non-uniform stiffness for each sector, as illustrated in Fig 15. More particularly, the stiffness of each blade is modified such that the square of the clamped blade eigenfrequencies matches with the mistuning identified experimentally. Table 3 gives the values of the geometric mistuning and the "clamping mistuning" for each blade. The geometry mistuning is defined here as the variation in the volume of the blade compared to the mean volume. Both mistunings are of the same order of magnitude but the frequency mistuning remains larger (up to $7 \%$ ). 


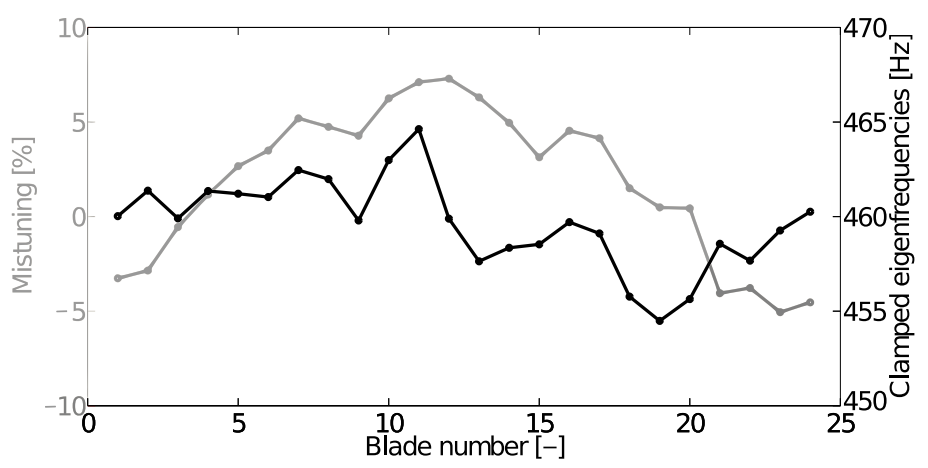

Figure 14: Mistuning identified experimentally (in gray) and clamped blade eigenfrequencies (in black).

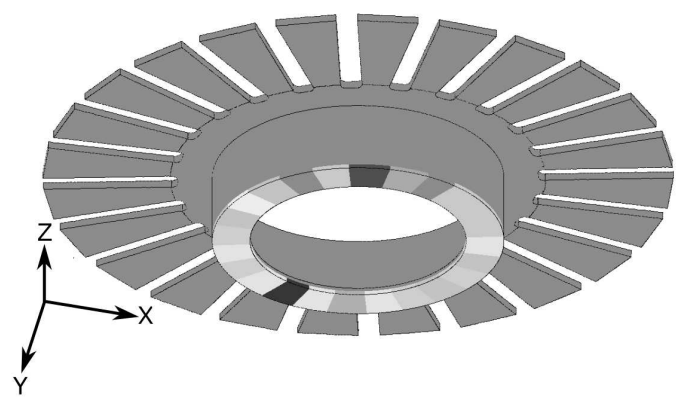

Figure 15: Non uniform clamping conditions.

The new mode-shapes are given in Fig. 16. A good correspondence is obtained for the first three modes. The MAC values between experimental and numerical mode-shapes are 1 for the three considered modes.

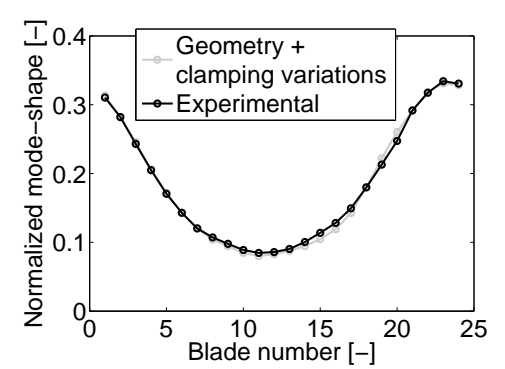

(a)

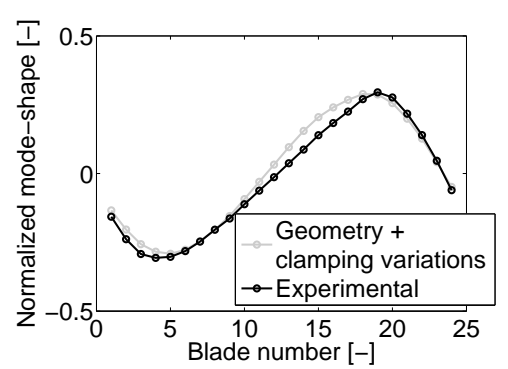

(b)

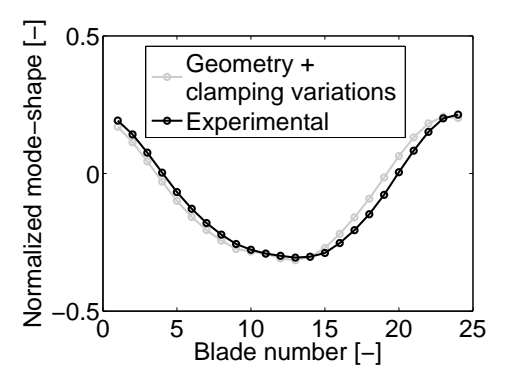

(c)

Figure 16: Mode-shapes corresponding to the first (a), second (b) and third (c) eigenfrequencies. 


\begin{tabular}{|c|c|c|}
\hline $\begin{array}{c}\text { Blade } \\
\text { number [-] }\end{array}$ & $\begin{array}{c}\text { Geometry } \\
\text { mistuning [\%] }\end{array}$ & $\begin{array}{c}\text { Frequency } \\
\text { mistuning [\%] }\end{array}$ \\
\hline 1 & 0.95 & -3.40 \\
\hline 2 & 1.02 & -3.37 \\
\hline 3 & 0.41 & -0.47 \\
\hline 4 & 0.40 & 0.45 \\
\hline 5 & 0.79 & 1.72 \\
\hline 6 & 0.77 & 2.75 \\
\hline 7 & 0.52 & 3.67 \\
\hline 8 & 0.05 & 3.63 \\
\hline 9 & 0.37 & 4.34 \\
\hline 10 & 0.08 & 5.03 \\
\hline 11 & -0.74 & 4.97 \\
\hline 12 & -0.56 & 7.26 \\
\hline 13 & -0.23 & 6.94 \\
\hline 14 & -0.49 & 5.45 \\
\hline 15 & -0.32 & 3.60 \\
\hline 16 & -0.42 & 4.29 \\
\hline 17 & -0.74 & 4.16 \\
\hline 18 & -1.13 & 3.14 \\
\hline 19 & -0.81 & 2.72 \\
\hline 20 & -0.44 & 2.31 \\
\hline 21 & -0.40 & -3.53 \\
\hline 22 & 0.35 & -2.71 \\
\hline 23 & 0.23 & -4.81 \\
\hline 24 & 0.35 & -4.55 \\
\hline
\end{tabular}

Table 3: Geometry and frequency mistuning.

The modification of the clamping conditions enables to improve the correspondence between the modeshapes of the numerical model and the experimental data. The remaining error in blade mistuning using the updated numerical mode-shapes is $3 \%$. This work enables to conclude that the mistuning of blade can be determined experimentally, and this identified mistuning has two main sources: the geometry variations between the different sectors which are due to the manufacturing tolerances, and the clamping conditions which differ from the perfect clamping conditions usually used in the finite element models. 


\section{Conclusion}

The mistuning of an academic bladed structure has been identified experimentally by using the component mode mistuning method. A base excitation with an electrodynamic shaker was used to excite the structure in the frequency range of interest and the response was measured on each blade by use of a laser vibrometer.

The main source of mistuning for this structure is the geometric variations of each sector due to the manufacturing tolerances. Indeed, since the bladed disk is a one-piece structure, there is no welded connections between the blades and the disk and the material properties can be assumed to be uniform. An optical geometry measurement system was used in order to retrieve the exact profile of each blade. This enables to determine the mistuning of each blade due to variations in the geometry. Differences have been observed between the identified mistuning and the geometric mistuning. These differences may be attributed to non uniformities in the clamping conditions.

Both sources of mistuning have been quantified. It was found in this example that both geometric mistuning and clamping non uniformities are of the same order of magnitude (1.2 and $7 \%$ respectively). Depending of the structure, the relative importance between the different sources of mistuning can change.

The indirect mistuning induced by clamping conditions can be reduced by modifying the test set-up to tend to the perfect clamping conditions, for example by adding a steel plate at the center of the structure. It would allow reducing the effect of the clamping conditions, but would not be representative of actual boundary conditions. In practice, connections between the blades and the disk, wear during use, or material non uniformities are additional sources of indirect mistuning.

Since numerous factors induce mistuning in the structure, the experimentally identified mistuning can therefore differ from the actual mistuning of the structure in real conditions. Consequently, this work evidences the importance of characterizing the level of mistuning induced during the manufacturing and assembly processes and the interest of using probabilistic approaches since the knowledge of the exact mistuning is illusory. Probabilistic approaches enable to evaluate the sensitivity of the amplification of the forced response with respect to small changes in the considered mistuning.

\section{Acknowledgements}

The first author F. Nyssen is supported by a grant from the Belgian National Fund for Scientific Research (FNRS) which is gratefully acknowledged.

\section{References}

[1] M. P. Castanier, C. Pierre, Modeling and Analysis of Mistuned Bladed Disk Vibration: Current Status and Emerging Directions, Journal of Propulsion and Power (2) 384-396. doi:10.2514/1.16345 
[2] H. Irretier, Spectral Analysis of Mistuned Bladed-Disc Assemblies by Component Mode Synthesis, in: Proceedings of the ASME 9th Biennal Conference on Mechanical Vibration and Noise, American Society of Mechanical Engineers, Fairfield, NJ, USA, 1983, pp. 115-125.

[3] Z.-C. Zheng, F. R. Wang, Dynamic Analysis of Blade Groups Using Component Mode Synthesis, in: Proceedings of the ASME 10th Biennal Conference on Mechanical Vibration and Noise, American Society of Mechanical Engineers, Fairfield, NJ, USA, 1985, pp. 97-103.

[4] M. J. Kruse, C. Pierre, Forced Response of Mistuned Bladed Disks Using Reduced-Order Modeling, in: Proceedings of the 37th AIAA/ASME Structures, Structural Dynamics and Materials Conference, Reston, VA, USA, pp. 1938-1950.

[5] M. P. Castanier, G. Ottarsson, C. Pierre, A Reduced Order Modeling Technique for Mistuned Bladed Disks, Journal of Vibration and Acoustics (3) 439-447. doi:10.1115/1.2889743

[6] M.-T. Yang, J. H. Griffin, A Reduced-Order Model of Mistuning Using a Subset of Nominal System Modes, Journal of Engineering for Gas Turbines and Powerdoi:10.1115/1.1385197

[15] J. A. Judge, C. Pierre, S. L. Ceccio, Experimental Mistuning Identification in Bladed Disks Using a Component-ModeBased Reduced-Order Model, AIAA Journal (5) 1277-1287. doi:10.2514/1.41214

[16] D. M. Feiner, J. H. Griffin, Mistuning Identification of Bladed Disks Using a Fundamental Mistuning ModelPart I: Theory, Journal of Turbomachinery (1) 150-158. doi:10.1115/1.1643913

[17] D. M. Feiner, J. H. Griffin, Mistuning Identification of Bladed Disks Using a Fundamental Mistuning Model Part II: Application, Journal of Turbomachinery (1) 159-165. doi:10.1115/1.1643914

[18] M. P. Mignolet, C.-C. Lin, Identification of Structural Parameters in Mistuned Bladed Disks, Journal of Vibration and Acoustics (3) 428-438. doi:10.1115/1.2889742.

[19] F. Pichot, F. Thouverez, L. Jezequel, E. Seinturier, Mistuning Parameters Identification of a Bladed Disk, Key Engineering Materials 123-132doi:10.4028/www.scientific.net/KEM.204-205.123.

[20] N. E. Kim, J. H. Griffin, System Identification in Higher Modal Density Regions of Bladed Disks, in: Proceedings of the 8th National Turbine Engine High Cycle Fatigue Conference, Dayton, OH, USA, 2003, pp. 1.68-1.82.

[21] S.-H. Lim, M. P. Castanier, C. Pierre, Vibration Modeling of Bladed Disks Subject to Geometric Mistuning and Design Changes, in: 45th AIAA/ASME/ASCE/AHS/ASC Structures, Structural Dynamics, and Materials Conference, Palm 
Springs, California, 2004, pp. 1-20.

[22] F. Pichot, D. Laxalde, J.-J. Sinou, F. Thouverez, J.-P. Lombard, Mistuning Identification for Industrial Blisks Based on the Best Achievable Eigenvector, Computers \& Structures (29-30) 2033-2049. doi:10.1016/j.compstruc.2006.08.022

[23] D. Laxalde, F. Thouverez, J.-J. Sinou, J.-P. Lombard, S. Baumhauer, Mistuning Identification and Model Updating of an Industrial Blisk, International Journal of Rotating Machinery 1-10doi:10.1155/2007/17289

[24] S. H. Song, M. P. Castanier, C. Pierre, System Identification of Multistage Turbine Engine Rotors, in: Proceedings of ASME Turbo Expo 2007, Montreal, Canada, 2007, pp. 1-14.

[25] A. C. Madden, M. P. Castanier, B. I. Epureanu, Reduced-Order Model Construction Procedure for Robust Mistuning Identification of Blisks, AIAA Journal (11) 2890-2898. doi:10.2514/1.37314

[26] A. Kaszynski, J. A. Beck, J. M. Brown, Uncertainties of an Automated Optical 3D Geometry Measurement, Modeling, and Analysis Process for Mistuned IBR Reverse Engineering, in: Proceedings of ASME Turbo Expo 2013, San Antonio, Texas, USA, 2013, pp. 1-9.

[27] F. Nyssen, M. Arnst, J.-C. Golinval, Experimental Modal Identification of Mistuning in an Academic Blisk And Comparison With The Blades Geometry Variations, Proceedings of the ASME Turbo Expo 2015.

[28] M.-T. Yang, J. H. Griffin, A Normalized Modal Eigenvalue Approach for Resolving Modal Interaction, Journal of Engineering for Gas Turbines and Power (3) 647-650. doi:10.1115/1.2817033

[29] LMS Samtech. URL http://www.plm.automation.siemens.com/frbe/products/lms/samtech/index.shtml

[30] B. Peeters, H. Van der Auweraer, P. Guillaume, J. Leuridan, The PolyMAX frequency-domain method: a new standard for modal parameter estimation?, Journal of Shock and Vibration (3-4) 395-409.

[31] LMS Test.Lab: Siemens PLM Software URL http://www .plm . automation. siemens . com/frbe/products/lms/

[32] GOM

URL http://www.gom.com 